ARTICLE

\title{
Benchmarking of PHITS on Pion Production for Medium-Energy Physics
}

\author{
Norihiro MATSUDA ${ }^{1, *}$, Yosuke IWAMOTO ${ }^{1}$, Hiroshi IWASE ${ }^{2}$, \\ Yukio SAKAMOTO ${ }^{1}$, Hiroshi NAKASHIMA ${ }^{1}$ and Koji NIITA ${ }^{3}$ \\ ${ }^{1}$ Japan Atomic Energy Agency, 2-4 Shirakata, Tokai-mura, Naka-gun, Ibaraki, 319-1195, Japan \\ ${ }^{2}$ High Energy Accelerator Research Organization, 1-1 Oho, Tsukuba, Ibaraki, 305-0801, Japan \\ ${ }^{3}$ Research Organization for Information Science \& Technology, 2-4 Shirakata, Tokai-mura, Naka-gun, Ibaraki, 319-1106, Japan
}

\begin{abstract}
Bench marking calculation for PHITS was performed on pion production cross sections for $\mathrm{C}, \mathrm{Al}, \mathrm{Cu}$ and $\mathrm{Pb}$ target irradiated by $730-\mathrm{MeV}$ protons at wide angles in the interval 15 to 150 degrees. The experimental double differential cross sections were compared with the calculation results calculated by PHITS. As results, the PHITS calculations gave quite satisfactory results on the whole.
\end{abstract}

KEYWORDS: benchmarking calculation, pion cross section, PHITS

\section{Introduction}

Particle and Heavy Ion Transport code System, PHITS, ${ }^{1)}$ which can treat interaction and transport phenomena for particles and heavy ions, has been used for various purposes in a broad range of fields: radiation shielding design, dosimetry, radiotherapy and space science. Many validations for PHITS are required and performed in individual cases, as well as pion production. Benchmarking analysis has been carried out in the Hadron Shower Simulation workshop ${ }^{2)}$ held at 2006 (HSS06) at Fermi National Accelerator Facility. The purpose of the workshop is to lead to better understanding and simulation of hadronic showers relevant to hadron calorimetry at the International Linear Collider (ILC) ${ }^{3)}$ and Large Hadron Collider (LHC), ${ }^{4)}$ neutrino fluxes from pionic decay and atmospheric showers. One of the benchmarking problems, high-energy pion production (energy spectra and momenta in the forward angle) for simulation of neutrino fluxes, was performed and reproduced very well by PHITS. And the other codes attended the workshop also provided good accuracy: MARS and FLUKA, slightly underestimation: LAQGSM03, and overestimation: DPMJET-III and GEANT4, on the whole. As a result of the benchmarking at the workshop, it was found that agreement between the simulation codes including PHITS and experimental data is quite reasonable in many cases.

In this study, verification for PHITS ver. 2.24 was done in medium-energy pion production through a benchmarking. Benchmark calculation is on pion production cross sections for some solid targets irradiated by $730-\mathrm{MeV}$ protons. The accuracy is extremely important from the perspective of some of the basic data for medium-energy physics.

*Corresponding author, E-mail: matsuda.norihiro@jaea.go.jp

\section{Benchmark Calculation}

\section{Experimental Data}

Experimental data ${ }^{5)}$ at the Lawrence Radiation Laboratry was taken up as a benchmarking problem for pion (plus and minus) production cross sections by $730-\mathrm{MeV}$ protons accelerated by 184-in. cyclotron on Carbon, Aluminum, Copper and Lead solid targets at wide angles in the interval 15 to 150 degrees. The experimental setup is shown in Fig. 1. The thickness of the target was varied for different target materials. Target densities are listed in Table 1. The solid targets were either $7.5 \mathrm{~cm}$ or $10 \mathrm{~cm}$ in diameter in the original paper. While the diameter of the target is very large compared with the thickness, the correction factor was not specified in this matter.

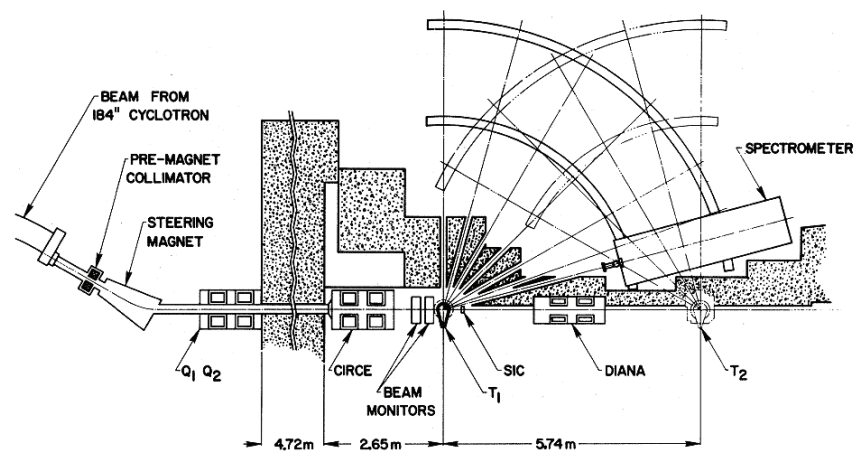

\# This figure is taken from Phys. Rev. D6, 11 (1972) by D. R. F. Cochran, et. al.

Fig. 1 Floor plan of beam line and spectrometer. Target position, $T_{1}$ is at angles of 15 to 90 degrees. $T_{2}$ is at 90 to 150 degrees. The distance from the target to the spectrometer is about $3.5 \mathrm{~m}$. In the calculation, the diameter of the target was decided $7.5 \mathrm{~cm}$ except for 90 degree. At 90 degree, the diameter was also decided the same thickness as the target densities. 
Table 1 Target densities

\begin{tabular}{cc}
\hline Material & $\begin{array}{c}\text { Density } \\
\left(\mathrm{g} \mathrm{cm}^{-2}\right)\end{array}$ \\
\hline $\mathrm{C}$ & 1.10 \\
$\mathrm{Al}$ & 0.97 \\
$\mathrm{Cu}$ & 0.97 \\
$\mathrm{~Pb}$ & 1.90 \\
\hline
\end{tabular}

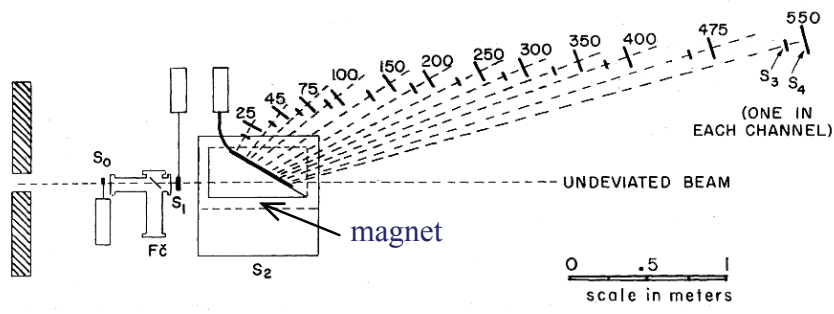

\# This figure is taken from Phys. Rev. D6, 11 (1972) by D. R. F. Cochran, et. al.

Fig. 2 Spectrometer consisting of a bending magnets, and an array of 12 sets of counter telescopes $\left(\mathrm{S}_{3}\right.$ and $\left.\mathrm{S}_{4}\right)$. Energies in the interval 25 to $550 \mathrm{MeV}$ can be measured using the spectrometer.

On the condition, the intensities of secondary pions around 90 degree will be obviously over-decreased by the shielding effect of the target itself. We estimated this issue, and found the different tendencies between 90 degree and the other angles. Therefore, we assumed the numerical cross section data in the paper around 90 degree are corrected. In the calculation, the diameter of the target was decided $7.5 \mathrm{~cm}$, except for 90 degree. At the 90 degree, the diameter was also decided the same thickness as the target densities, listed in Table 1 that we could consider the self-shielding effect is negligible small. The pion spectrometer consists of a bending magnet and 12 sets of scintillation counters. The spectrometer can measure at twelve energies in the interval 25 to $550 \mathrm{MeV}$. The pion spectrometer is shown in Fig. 2.

\section{Calculation Geometry}

Calculation geometry was precisely reproduced such as, the target thickness and diameter, and the distance from the target to the spectrometer, except for 90 degree. At the 90 degree, the diameters were used the same thicknesses as the each target densities, listed in Table 1, respectively.

\section{Calculation Results}

Calculation results are compared with the experimental data on pion $(\pi+$ and $\pi-)$ production cross sections. The PHITS calculations were performed using two physics mod$\mathrm{el}, \mathrm{JAM}^{6)}$ and $\mathrm{JQMD}^{7)}$ codes, which describe intranuclear cascade phase. The comparison of the cross sections given for the following target materials: $\mathrm{C}, \mathrm{Al}, \mathrm{Cu}$ and $\mathrm{Pb}$, selected for the following angles: 15, 30, 60, 90, 120 and 150 degrees, and incident energy of $730 \mathrm{MeV}$ are presented in Figs. 3-10.

The calculation results by PHITS (JAM and JQMD) of pion production cross sections for Carbon (light nuclei) were

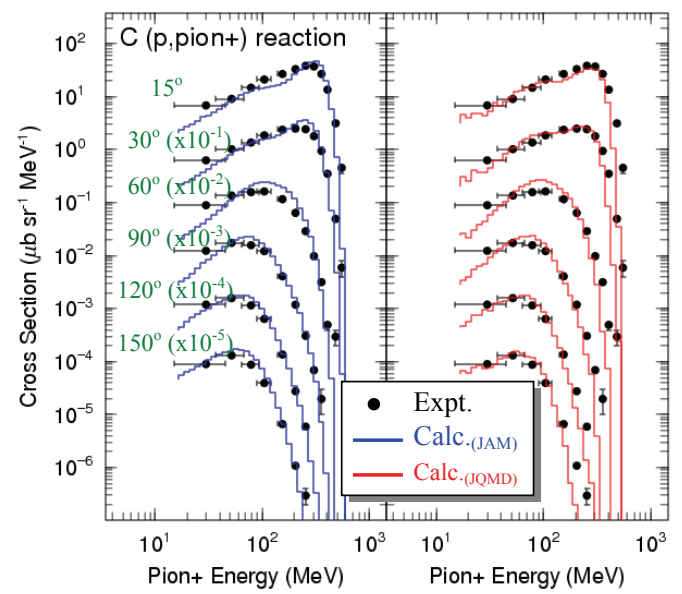

Fig. 3 Pion plus production cross section for $730-\mathrm{MeV}$ protons on Carbon target. Blue solid lines are calculated by JAM in the PHITS. Red solid lines are JQMD results.

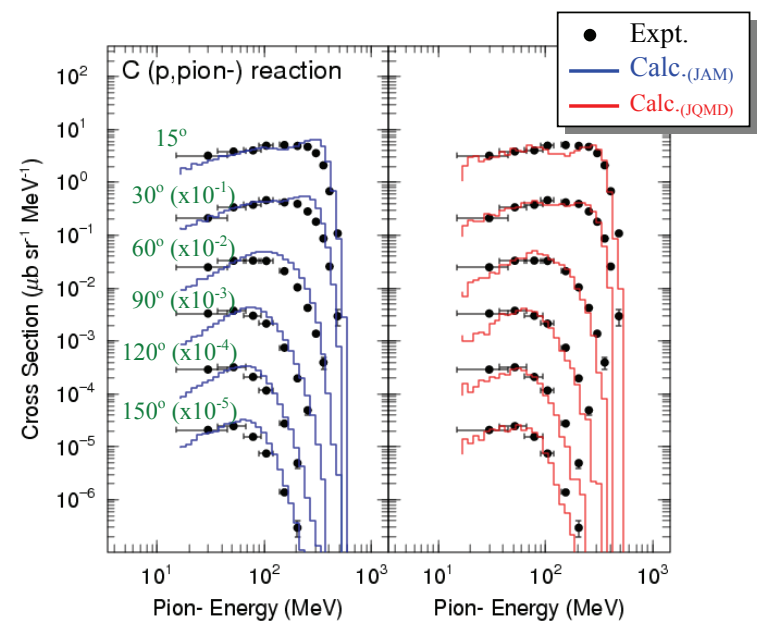

Fig. 4 Pion minus production cross section for $730-\mathrm{MeV}$ protons on Carbon target. Blue solid lines are calculated by JAM in the PHITS. Red solid lines are JQMD results.

excellent agreement with the experimental data on the whole. The curves for $\pi^{+}$and $\pi^{-}$meson production cross section, which have strong forward peaking shown in Figs. 3 and 4, were also reproduced by the JAM and JQMD calculations.

The cross sections for Aluminum, Copper and Lead (heavy nuclei) were also compared between the PHITS calculation and experimental data. The Al cross sections, shown in Figs. 5 and 6, were good agreement, except for pion+ meson production in forward angle. The Copper double differential cross sections were well reproduced by the PHITS calculation on the whole. In the forward angle, however, double-peak shape of the cross sections calculated by the JAM code were appeared for pion+ and pion-, and slightly appeared in the JQMD results. The same tendency was also shown the cross sections for Lead, in Figs. 9 and 10. For the heavier nuclei, PHITS (the JAM and the JQMD codes) were not so good agreement with the experimental data. Especially, pion minus cross sections calculated by the JQMD code 


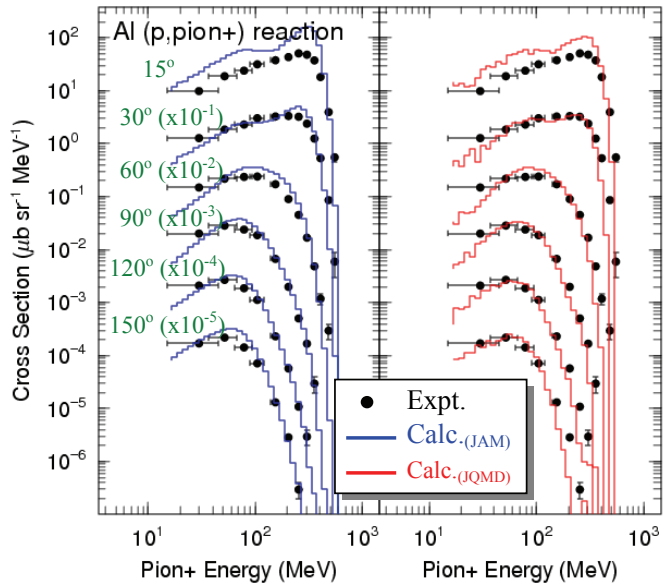

Fig. 5 Pion plus production cross section for $730-\mathrm{MeV}$ protons on Aluminum target. Blue solid lines are calculated by JAM in the PHITS. Red solid lines are JQMD results.

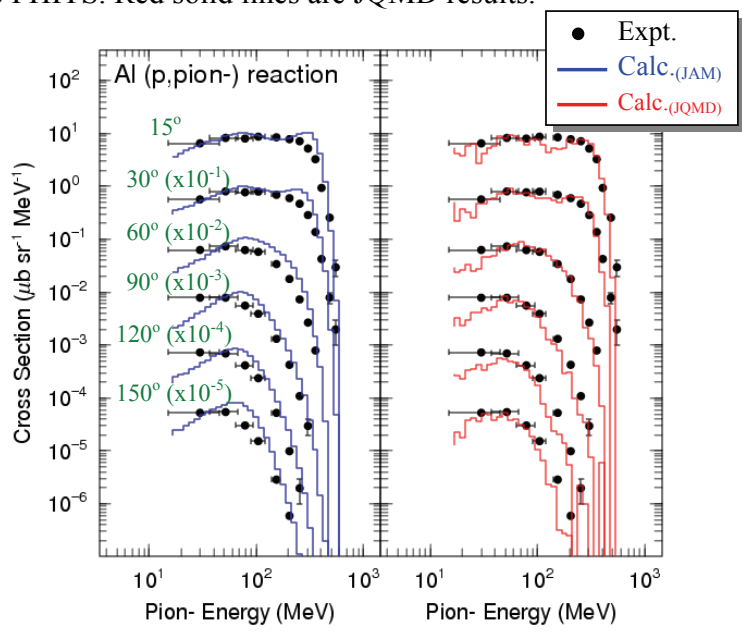

Fig. 6 Pion minus production cross section for $730-\mathrm{MeV}$ protons on Aluminum target. Blue solid lines are calculated by JAM in the PHITS. Red solid lines are JQMD results.

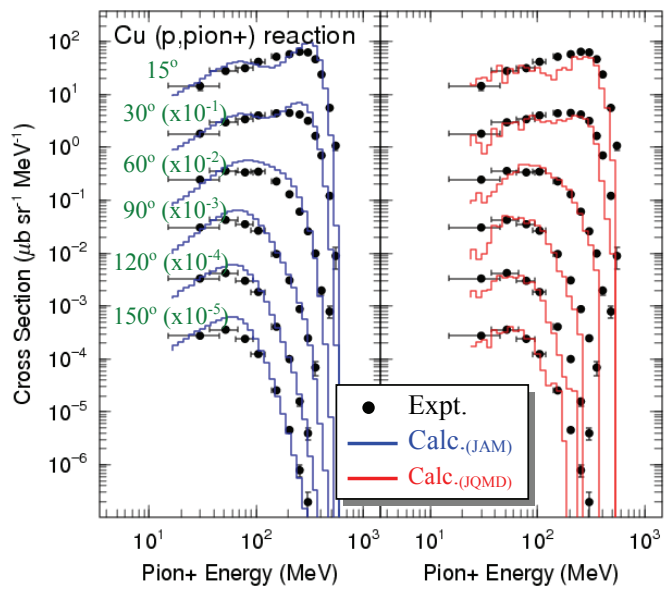

Fig. 7 Pion plus production cross section for $730-\mathrm{MeV}$ protons on Copper target. Blue solid lines are calculated by JAM in the PHITS. Red solid lines are JQMD results.

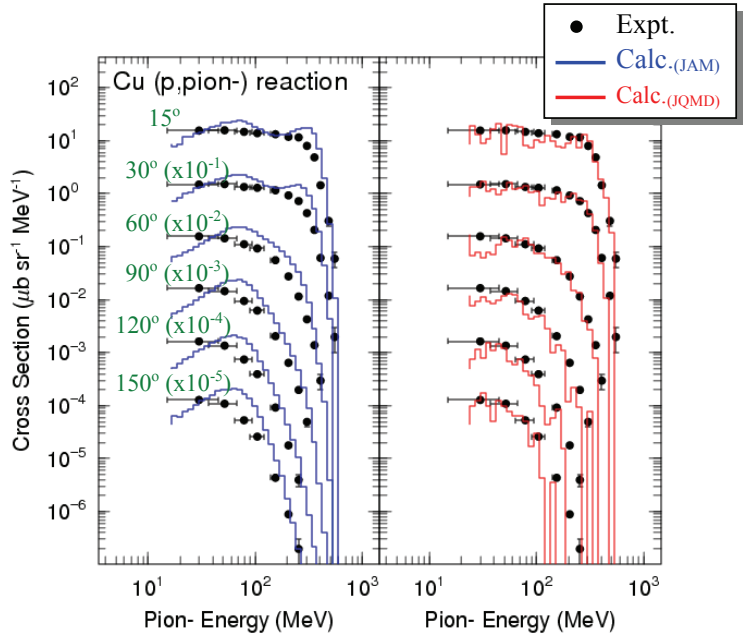

Fig. 8 Pion minus production cross section for $730-\mathrm{MeV}$ protons on Copper target. Blue solid lines are calculated by JAM in the PHITS. Red solid lines are JQMD results.

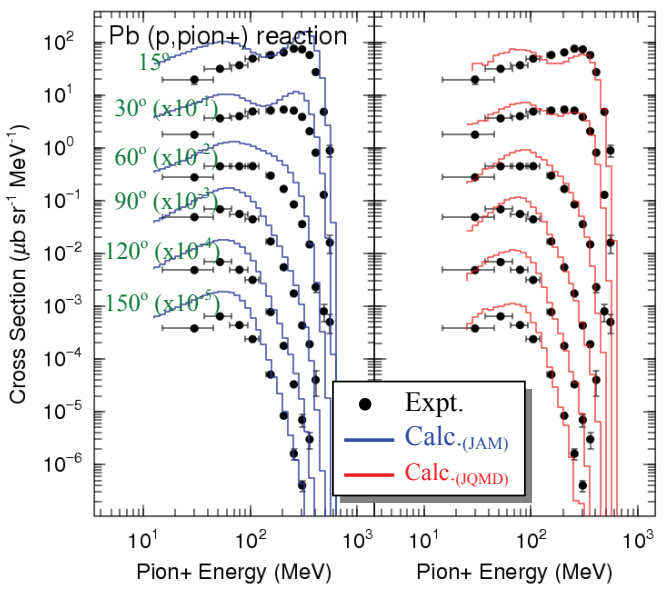

Fig. 9 Pion plus production cross section for $730-\mathrm{MeV}$ protons on Lead target. Blue solid lines are calculated by JAM in the PHITS. Red solid lines are JQMD results.

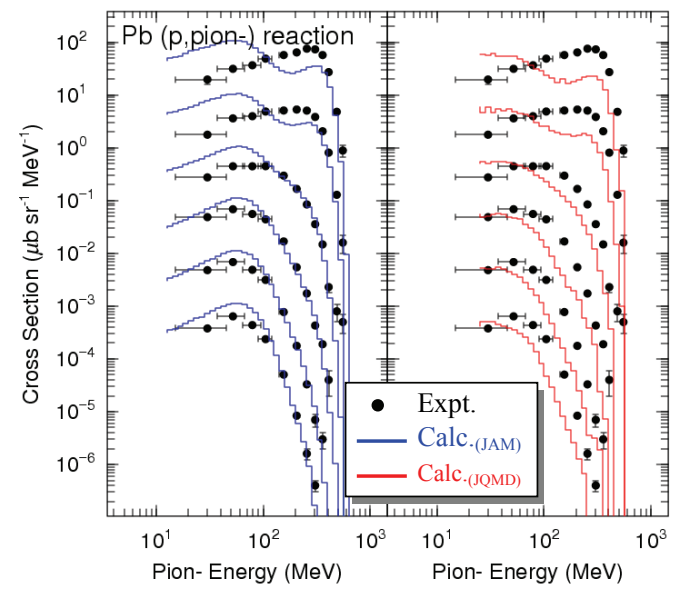

Fig. 10 Pion minus production cross section for $730-\mathrm{MeV}$ protons on Lead target. Blue solid lines are calculated by JAM in the PHITS. Red solid lines are JQMD results. 
were underestimated the experimental data. The physical mechanism of the double-peak shape is not clear, but might be related to the reabsorption process of pions in nucleus. In this sense, the JAM and the JQMD codes have almost the same performance on pion production, because the production cross section is almost the same and the same absorption cross section is used in PHITS.

\section{Conclusion}

Benchmarking calculation on pion production cross sections for medium-energy physics was performed using PHITS. The experimental data were used given for the following target materials: $\mathrm{C}, \mathrm{Al}, \mathrm{Cu}$ and $\mathrm{Pb}$, selected for the following angles: 15, 30, 60, 90, 120 and 150 degrees, and incident energy of $730 \mathrm{MeV}$. The cross sections calculated by JAM and JQMD in PHITS were compared with the experimental data. As results, the PHITS calculations gave quit satisfactory results on the whole, except for the heavier nuclei. The JAM and the JQMD codes had almost the same description on pion production, which had been well verified compared with the experimental data of nucleon-nucleon collisions. However, these codes do not include the elastic collisions between pion and nucleon in intra-nuclear cascade processes, which may become more important in hevier system. Therefore more study on the treatment for the reabsorption process of pion in nuclei will be needed in order to perform the accuracy improvement of these codes in PHITS.

\section{References}

1) K. Niita, T Sato, H. Iwase, H. Nose, H. Nakashima, L. Sihver, "PHITS -a particle and heavy ion transport code system," $R a$ diat. Meas., 41, 1080-1090 (2006).

2) N. V. Mokhov, S. I. Striganov, "Hadronic shower code inter-comparison and verification," American Institute of Physics Conf. Proc., 896, 112-122, Hadronic Shower Simulation Workshop, Batavia, IL, USA, Sept. 6-8, 2006, (2007); http://conferences.fnal.gov/hss06.

3) Reference Design Report, ILC-Report-2007-001, (2007); $\mathrm{http}: / /$ www.linearcollider.org.

4) K. M. Potter, The large hadron collider (LHC) project of CERN, LHC project report, 36, European Organization for Nuclear Research (CERN) (1996).

5) D. R. F. Cochran, P. N. Dean, P. A. M. Gram, E. A. Knapp, E. R. Martin, D. E. Nagle, R. B. Perkins, W. J. Shlaer, H. A. Thiessen, E. D. Theriot, "Production of charged pions by $730-\mathrm{MeV}$ protons from hydrogen and selected nuclei," Phys. Rev., D6, 11, 3085-3116 (1972).

6) Y. Nara, N. Otsuka, A. Ohnishi, K. Niita, S. Chiba, "Relativistic nuclear collisions at $10 A \mathrm{GeV}$ energies from $p+\mathrm{Be}$ to $\mathrm{Au}+\mathrm{Au}$ with the hadronic cascade model," Phys. Rev., C61, 024901 (1999).

7) K. Niita, S. Chiba, T. Maruyama, H. Takada, T. Fukahori, Y. Nakahara, A Iwamoto, " Analysis of the $(N, x N$ ') reactions by quantum molecular dynamics plus statistical decay model," Phys. Rev., C52, 2620-2635 (1995).

8) S. Furihata, "Statistical analysis of light fragment production from medium energy proton-induced reactions," Nucl. Instr. Meth. Phys. Res., B171, 251-258 (2000). 